ISSN 1112-9867

http://www.jfas.info

\title{
CONTRIBUTION TO THE EXPERIMENTAL STUDY OF THE HYDRAULIC JUMP IN TRAPEZOIDAL CHANNEL WITH POSITIVE SLOPE
}

\author{
A. Ghomri ${ }^{* 1,2}$, F. Riguet ${ }^{1,2}$, S. Khechana ${ }^{2}$ \\ ${ }^{1}$ Laboratoire de Recherche en Hydraulique Souterraine et de Surface, Université Biskra \\ ${ }^{2}$ Laboratoire d'exploitation et de valorisation des ressources energétiques sahariènnes, \\ université d'El Oued
}

Received: 27 October 2017 / Accepted: 17 December 2017 / Published online: 01 January 2018

\begin{abstract}
The purpose of this study is to study experimentally the hydraulic jump evolving in a symmetric trapezoidal channel with a positive slope, requires the use of an experimental protocol, and to find experimental relations linking the characteristics of the formed projection. The experimental study investigated the variation of the relative height $S=s / h_{1}$ of the threshold as a function of the Froude F1 number of the incident flow, for five distinct values of the slope of the channel.
\end{abstract}

Keywords : Hydraulic jump, trapezoidal channel, damping basin, trapezoidal channel with positive slope, suddenly varied flow.

Author Correspondence, e-mail: alighomri@yahoo.fr

doi: http://dx.doi.org/10.4314/jfas.v10i1.14

\section{INTRODUCTION}

L'étude du ressaut hydraulique évoluant dans un canal trapézoïdal symétrique à un angle d'ouverture de $72,68^{\circ}$ à pente positive, nécessite l'utilisation d'un protocole expérimental, afin d'estimer d'une part le coefficient de correction $\mathrm{k}$ évoqué dans l'étude théorique, et d'autre part de trouver des relations 
empiriques liant les caractéristiques que l'on ne peut évaluer théoriquement.

Dans cette présente recherche nous allons aborder d'abord la description des essais effectués pour cette étude. Nous décrirons en premier lieu le protocole expérimental, constitué du canal trapézoïdal, des appareils et instruments utilisés pour la mesure des caractéristiques du ressaut hydraulique.

Nous passerons à la procédure expérimentale suivie par des essais en second lieu,et en dernier lieu nous exposerons enfin les résultats obtenus.

\subsection{PROTOCOLE EXPERIMENTAL}

Le canal de mesures (figure 1 et photo 1) qui nous a servi de banc d'essai est de section trapézoïdale, il est constitué essentiellement par une structure métallique rigide, sur laquelle est fixé latéralement, des deux cotés une série de 6 panneaux transparents en plexiglas permettant les prises de vue et observations. Ces panneaux sont reliés entre eux par collage au silicone. L'étanchéité est assurée par des joints en caoutchouc et par le silicone. Un vérin manuel est utilisé pour varier la pente du canal (photo 5).

Le banc d'essais est constitué d'un grand canal rectangulaire dans lequel est inséré un canal de mesure de section trapézoïdale, caractérisé par une largeur de base $20 \mathrm{~cm}$, de longueur $6 \mathrm{~m}$ et un angle d'inclinaison des parois latérales de $72,68^{\circ}$; relier à un bassin d'accumulation par le moyen d'une conduite circulaire de $115 \mathrm{~mm}$ de diamètre. Celle-ci est reliée à une boite métallique fermée (photo 2), sur laquelle est insérée une ouverture en tôle de section trapézoïdale (photo 3) débouchant dans le canal. Le rôle de ce dernier est de générer un écoulement incident à grande vitesse. La section de sortie de celle-ci est variable et sa hauteur correspondra à la hauteur initiale $\mathrm{h}_{1} \mathrm{du}$ ressaut.L'ensemble fonctionne en circuit fermé dans lequel et insérée une pompe axiale (photo 4) débitant jusqu'à 44 1/s.

Le réglage des débits volumes s'effectue par manipulation de la vanne et sont mesurés à l'aide d'un débitmètre à affichage numérique (voir photographies cidessous). 


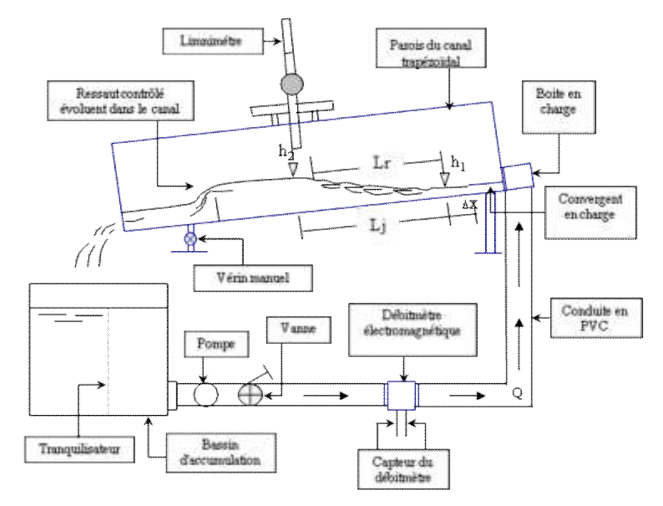

Fig.1. Schéma simplifié du canal de mesure de section trapézoïdale, ayant servi à l'expérimentation.
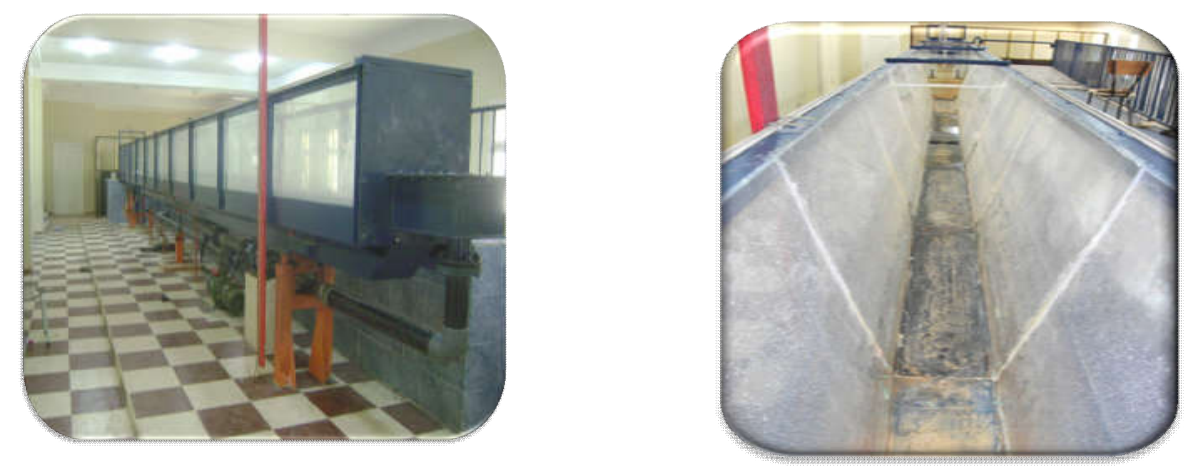

Photo 1: Photographie du canal de mesure utilisé.

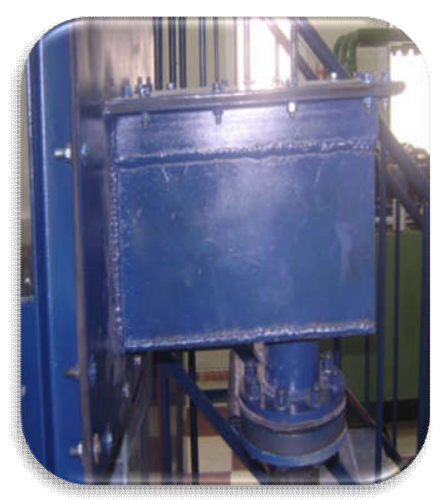

Photo 2: Photographie de la boite en charge.

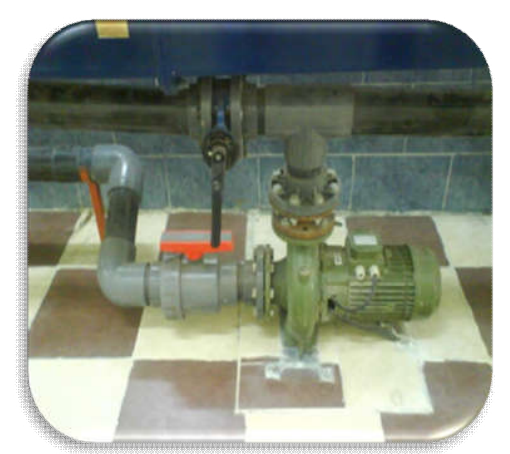

Photo 4: Photographie d'une Pompe centrifuge Photo 5: Photographie d'un vérin manuel
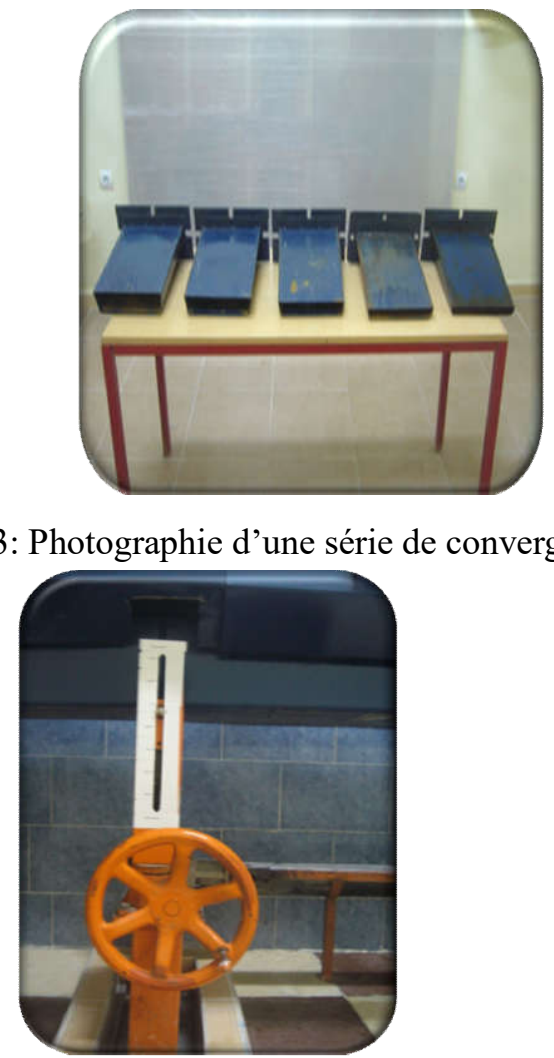

Photo 3: Photographie d'une série de convergent 


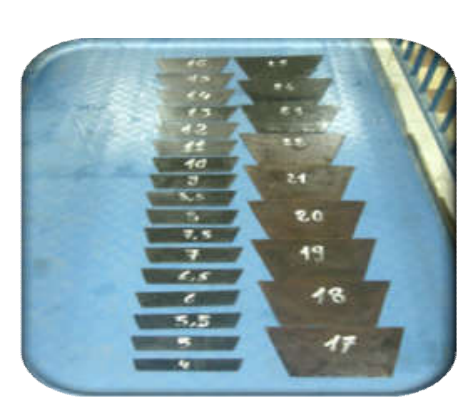

Photo6: Photographie de la série de seuil

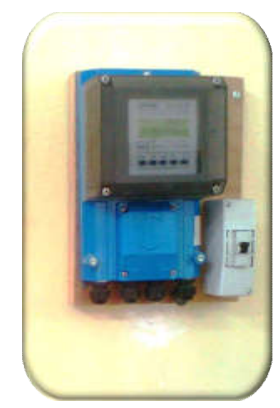

Photo7: convertisseur du débitmètre électromagnétique

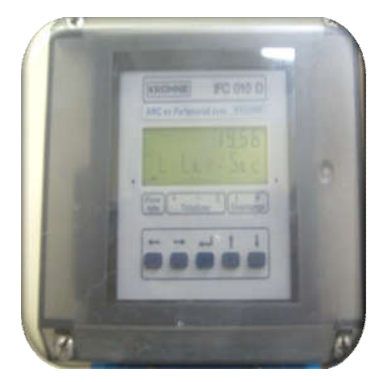

Photo 8: écran d'affichage des débits

\subsection{Description des essais}

L'étude expérimentale s'est intéressée au ressaut hydraulique contrôlé par seuil mince dans un canal trapézoïdal à pente positive. Les caractéristiques étudiées sont: le débit volume $\mathrm{Q}$, la hauteur initiale $h_{1}$, mesurée au pied de ressaut, la hauteur finale $h_{2}$, mesurée au droit de la longueur du rouleau, la hauteur s du seuil, l'angle d'inclinaison $\alpha$ du canal par rapport à l'horizontale, la longueur $\operatorname{Lr}$ du rouleau, mesurée à la fin de la formation du ressaut et la longueur $\mathrm{Lj}$ du ressaut, mesurée dans le même endroit de la hauteur finale $\mathrm{h}_{2}$.

Celles-ci sont formulées sous forme adimensionnelle pour composer les rapports suivants: le nombre de Froude $F_{1}$ de l'écoulement incident, le rapport $\mathrm{Y}=\mathrm{h}_{2} / \mathrm{h}_{1}$ des hauteurs conjuguées du ressaut, la hauteur relative $S=s / h_{1}$ du seuil, la longueur relative $\lambda j=L j / h_{1}$ du ressaut, et le coefficient de forme du canal $\mathrm{M}=\mathrm{mh}_{1} / \mathrm{b}$.

L'expérimentation a été menée sous cinq hauteurs initiales : $\mathrm{h}_{1}(\mathrm{~mm})=20 ; 30 ; 40 ; 50$ et $60 \mathrm{~mm}$. Pour chaque hauteur $\mathrm{h}_{1}$ choisie, on donne au canal cinq positions d'inclinaison d'angle $\alpha$ correspondant chacune a une valeur bien déterminée de la pente positive, de sorte que la tangente de l'angle d'inclinaison $\alpha$ par rapport à l'horizontale, prend les valeurs suivantes (en \%) : $0 ; 0,5 ; 1 ; 1,5$ et 2 . Une gamme assez pratique de nombres de Froude incident a été 
ainsi obtenue: $1<\mathrm{F}_{1}<14$.

Des seuils de différentes hauteurs's' ont été testés (photo 6), afin d'observer leur influence sur le contrôle du ressaut; vingt-sept seuils ont ainsi été confectionnés, dont la hauteur s varie entre $3 \mathrm{~cm}$ et $26 \mathrm{~cm}$.

Pour une hauteur $\mathrm{h}_{1}$ et pour une hauteur s et une position $\mathrm{x}$ du seuil de l'écoulement incident, l'augmentation du débit volume Q, provoque l'apparition d'un ressaut.

Le couple de valeur $\left(\mathrm{Q}, \mathrm{h}_{1}\right)$ permet le calcul du nombre de Froude $\mathrm{F}_{1}$ de l'écoulement incident. L'accroissement de $F_{1}$ entraîne à la fois le déplacement du ressaut vers l'aval ainsi l'augmentation de sa longueur du rouleau $\mathrm{Lr}$ et du ressaut $\mathrm{Lj}$. La distance $\Delta \mathrm{x}$ sur laquelle le ressaut s'étend augmente également et pour ramener le pied de celui-ci dans sa position initiale, c'est à dire à environ $5 \mathrm{~cm}$ de la sortie du convergent, la hauteur $\mathrm{s}$ du seuil situé à l'extrémité aval du canal doit être augmenté. Ainsi à chaque valeur du nombre de Froude $\mathrm{F}_{1}$ correspondent une valeur de la longueur $\mathrm{Lj}$ du ressaut et une valeur de la hauteur s du seuil.

Les photos 9 et 10 illustrent deux configurations typiques de ressaut contrôlé par seuil mince en canal trapézoïdale symétrique, respectivement à pente nulle et incliné d'un angle $\alpha$ par rapport à l'horizontal, pour la même hauteur initiale $\mathrm{h}_{1}=30 \mathrm{~mm}$ et à débit volume croissant.

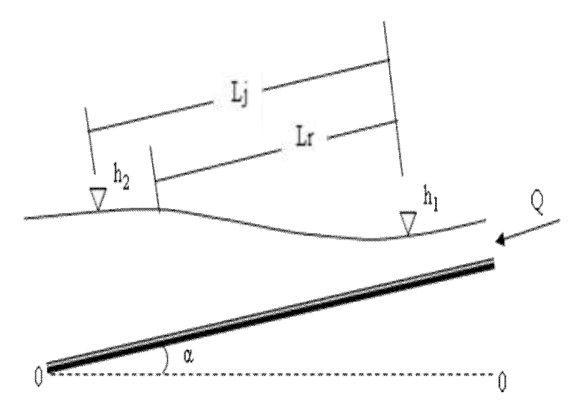

Figure 2: Schéma simplifier d'un ressaut hydraulique évoluant dans un canal à pente positive

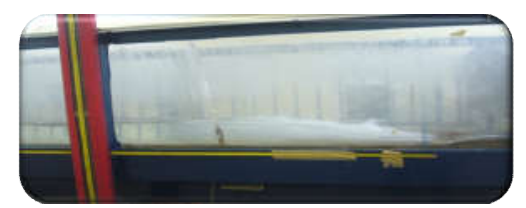

Photo 9:Photographie d'un ressaut hydraulique

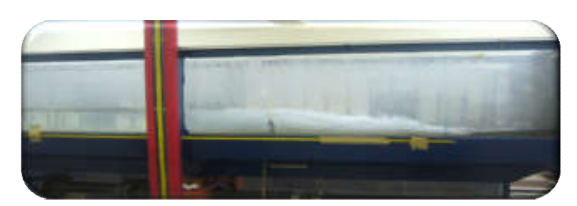

photo10:Photographie d'un ressaut hydraulique
Contrôlé à pente nulle $\mathrm{F}_{1}=6,63 ; \mathrm{s}=7 \mathrm{~cm}$; $\mathrm{Lj}=110 \mathrm{~cm} ; \mathrm{h}_{2}=13,7 \mathrm{~cm} ; \operatorname{tang}(\alpha)=0$ contrôlé à pente positive $\mathrm{F}_{1}=8,96$; $\mathrm{s}=17 \mathrm{~cm} ; \mathrm{Lj}=164 \mathrm{~cm} \quad \mathrm{~h}_{2}=20,8 \mathrm{~cm} ; \operatorname{tang}(\alpha)=0,01$ 
Pour obtenir les résultats expérimentaux, nous avons suivis les étapes suivantes:

Mesure de l'angle d'inclinaison $\alpha$ par rapport à l'horizontal;

Mesure de la longueur Lr du rouleau;

Mesure de la longueur Lj du ressaut;

Mesure de la hauteur géométrique s du seuil;

$$
F_{1}=\sqrt{\frac{Q^{2}[b(1+2 M)]}{g\left[b h_{1}(1+M)\right]^{3}}}
$$

Mesure de la hauteur conjuguée aval $h_{2}$ du ressaut;

Mesure du débit volume ;

Calcul du nombre de Froude $\mathrm{F}_{1}$ de l'écoulement incident, par application de la relation

\section{RESULTS ET DISCUSSION}

Les figures (3-7) montrent la variation de la hauteur relative $s / h_{1}$ du seuil en fonction du nombre de Froude $F_{1}$, pour cinq angles d'inclinaison $\alpha$ distincts, tels que :tang $(\alpha)=0.00,0.005,0.01,0.015,0.02$.

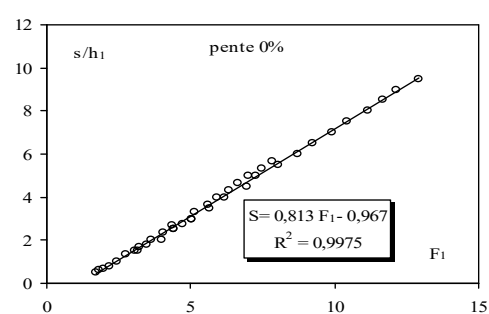

Figure 3 : Variation du rapport $\mathrm{s} / \mathrm{h}_{1}$ en function du nombre de Froude $F_{1}$,pour $\operatorname{tang}(\alpha)=0$.

o) points expérimentaux

\section{(一) Courbes d'ajustement}

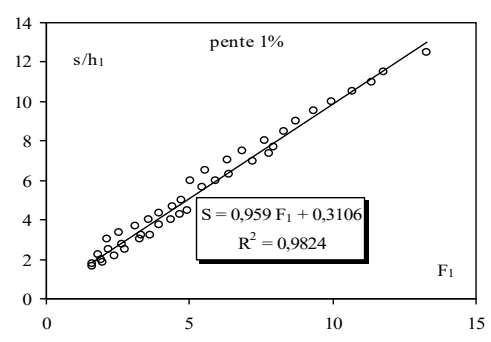

Figure 5: Variation du rapport $\mathrm{s} / \mathrm{h}_{1}$ en fonction du nombre de Froude $\mathrm{F}_{1}$, pour $\operatorname{tang}(\alpha)=0,01$. (o) Points expérimentaux (-) Courbesd'ajustement

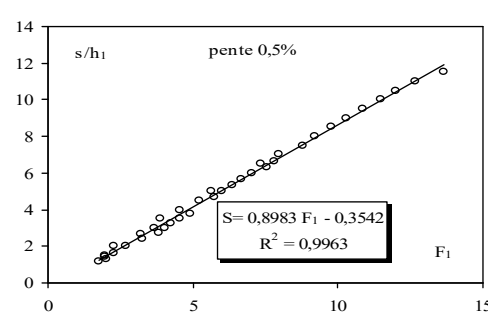

Figure 4: Variation du rapport $\mathrm{s} / \mathrm{h}_{1}$ en fonction du nombre de Froude $F_{1}$,pour $\operatorname{tang}(\alpha)=0,005$ o) points expérimentaux (-) Courbes d'ajustement

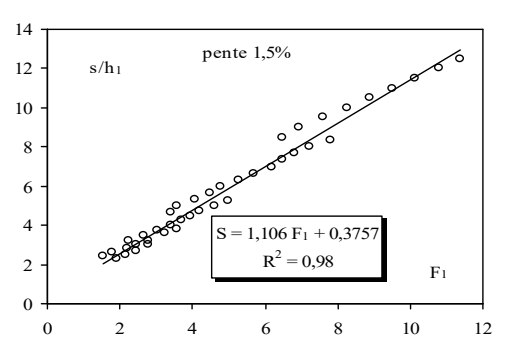

Figure 6 : Variation du rapport $\mathrm{s} / \mathrm{h}_{1}$ en fonction du nombre de Froude $F_{1}$, pour tang $(\alpha)=0,015$.(o) Points Expérimentaux (-) Courbes d'ajustement 


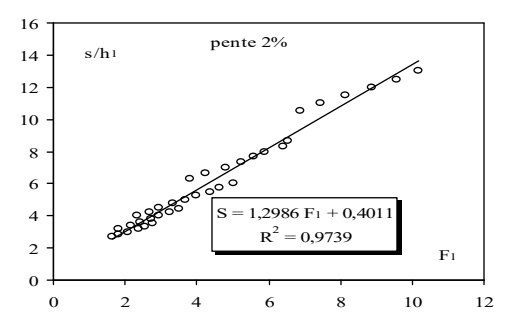

Figure 7 : Variation du rapport $\mathrm{s} / \mathrm{h}_{1}$ en fonction

du nombre de Froude $F_{1}$, pour $\operatorname{tang}(\alpha)=0,02$.

(o) points expérimentaux ; (-) Courbes d'ajustement

Selon les figures( 3-7), l'ajustement des mesures expérimentales a montré que la variation du rapport s/ $\mathrm{h}_{1}$ en fonction du nombre de Froude $\mathrm{F}_{1}$ de l'écoulement incident suit une loi de type linéaire de la forme $\mathrm{s} / \mathrm{h}_{1}=\mathrm{a}\left(\mathrm{F}_{1}\right)+\mathrm{b}$.

Le tableau 1 représente les paramètres a et $\mathrm{b}$ pour les cinq valeurs de pente considérées.

Tableau 1 : Valeurs des paramètres a et $b$.

Ce tableau montre clairement que les paramètres a et $\mathrm{b}$ sont fonction de la pente du canal et augmente progressivement avec l'augmentation de tang $(\alpha)$.

Les figures 8 et 9 montre l'ajustement statistique des couples de valeurs (a, tang $(\alpha))$ et $(b$, tang $(\alpha))$ par la méthode des moindre carrés.

Tableau 1 : Valeurs des paramètres a et $b$.

\begin{tabular}{|l|l|l|}
\hline \multicolumn{1}{|c|}{$\operatorname{tang}(\alpha)$} & $\mathrm{a}$ & $\mathrm{b}$ \\
\hline 0,000 & 0,81 & $-0,97$ \\
\hline 0,005 & 0,89 & $-0,35$ \\
\hline 0,010 & 0,96 & - \\
\hline 0,015 & 1,11 & 0,38 \\
\hline 0,020 & 1,30 & 0,40 \\
\hline
\end{tabular}
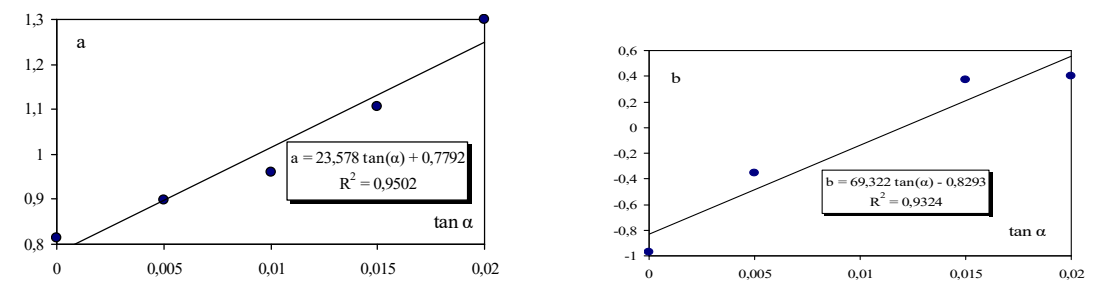

Figure 8 : Variation du paramètre 'a' en fonction de $\operatorname{tang}(\alpha)$.
Figure 9 : Variation du paramètre 'b' en fonction de $\operatorname{tang}(\alpha)$. 


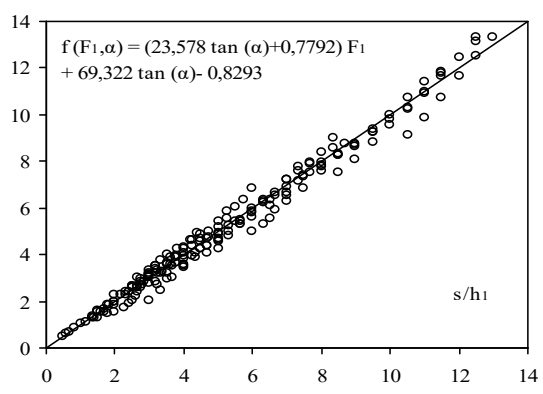

Figure 10. Variation de la relation $\mathrm{f}\left(\mathrm{F}_{1}, \alpha\right)$ en fonction de la hauteur relative $\mathrm{s} / \mathrm{h}_{1}$ du seuil. (o) Points expérimentaux. (-) Première bissectrice d'équation: $\mathrm{f}\left(\mathrm{F}_{1}, \alpha\right)=\mathrm{s} / \mathrm{h}_{1}$.

La figure 10 montre que les points se répartissent de manière quasi uniforme autour de la première bissectrice.

Les figures 8 et 9 montrent clairement que la variation de a et de ben fonction de la pente $(\operatorname{tang}(\alpha))$ du canal suit une loi linéaire pour les deux paramètres, selon les relations suivantes:

$$
\begin{aligned}
& \mathrm{a}=23,578 \operatorname{tang}(\alpha)+0,7792 ; \\
& \mathrm{b}=69,322 \operatorname{tang}(\alpha)-0,8293 ;
\end{aligned}
$$

En remplaçant les paramètres $a$ et $b$ par leurs expressions respectives dans la relation $s / h_{1}=a\left(F_{1}\right)+b$ on obtient la relation générale : s/h $\mathbf{h}_{1}=(23,578 \operatorname{tang}(\alpha)+0,7792) F_{1}+69,322 \operatorname{tang}(\alpha)-0,829$

$$
\text { avec } 1,52<\mathrm{F}_{1}<13,64 ; \quad 0 \leq \operatorname{tang}(\alpha) \leq 0.02
$$

La figure 10 confirme que la relation (3) représente un assez bon ajustement pour la détermination de la hauteur relative $s / h_{1}$ du seuil, connaissant le nombre de Froude incident $F_{1}$, et l'inclinaison $(\alpha)$ du canal.

\section{CONCLUSION}

Cette présente recherche a été consacrée à l'étude expérimentale du ressaut hydraulique dans un canal de section droite trapézoïdal à pente positive variable. Nous avons décrit dans un premier temps, le modèle physique qui nous a servi de banc d'essai, ainsi que les instruments et l'appareillage utilisé pour la mesure des caractéristiques du ressaut hydraulique, moyennant quelques schémas et des photographies. Dans un second temps, nous avons expliqué la procédure expérimentale suivie pendant les essais. Passant en troisième temps aux résultats expérimentaux, cinq (05) hauteurs initiales h1 ont été testées vingt (20) seuils s de hauteurs différentes et cinq (05) inclinaisons du canal par rapport à l'horizontale. Une large gamme des nombres de Froude incidents a été ainsi obtenue. L'étude expérimentale s'est intéressée, à la 
variation de la hauteur relative $S=s / h_{1}$ du seuil en fonction du nombre de Froude $F_{1}$ de l'écoulement incident, pour cinq valeurs distinctes de la pente du canal. Cinq nuages de points distincts, correspondant chacun à une valeur bien déterminée de la pente du canal. L'ajustement des mesures expérimentales a montré que la variation du rapport $\mathrm{S}$ en fonction du nombre de Froude $F_{1}$ de l'écoulement incident suit une loi de type linéaire de la forme $\mathrm{s} / \mathrm{h}_{1}=\mathrm{a}\left(\mathrm{F}_{1}\right)+\mathrm{b}$, régissant le ressaut hydraulique qui s'évolue en canal trapezoidal à pente posirive.

\section{REFERENCES}

[1] Achour, B., Debabeche, M., (2003), Ressaut contrôlé par seuil dans un canal profilé en U, J. Hydraulic Research, vol. 41, N0.3,pp.319-325.

[2] Achour, B., Debabeche, M., (2003), Control of Hydraulic jump by sill in a triangular channel, J. Hydraulic Research, Vol. 41, No. 3, pp. 97-103

[3] Anderson, V., M. (1978). Undular hydraulic jump, Proc., ASCE, J. Hydraulic Division, Vol. 104(HY8), pp. 1185-1188, discussion 1979, 105 (HY9), 1208-1211, 1980,106(HY7), 1252-1254.

[4] Debabeche, M., (2003), Le ressaut hydraulique dans les canaux prismatiques, Doctorat d'état, Département d'Hydraulique, Université de Biskra.

[5] Debabeche, M., (2008), Les écoulements brusquement variés, cours de 1ère année magister, polycopié, Université de Biskra.

[6] Debabeche, M., Achour, B., Effect of sill in the hydraulic jump in a triangular channel, J. Hydraulic Research Vol. 45, No. 1, (2007), pp. 135-139.

[7] Debabeche, M., Cherhabil, S., Hafnaoui, A. et Achour, B. (2009), Hydraulic jump in a sloped triangular channel, Can. J. Civ; Eng. 36: 2009, 655-658

[8] Ghomri, A., Debabeche, M., Riguet, F., Etude semi théorique du ressaut hydraulique évoluant en canal profilé en $U$, à fond rugueux, Revues des sciences fondamentales et appliquées, centre universitaire d'Oued Souf, $\mathrm{N}^{\mathrm{0}} 1$, 2009, pp. 41-56.

[9] Ghomri, A., Debabeche, M., Riguet, F., Etude expéimentale du ressaut hydraulique évoluant dans un canal en $\mathrm{U}$ à fond rugueux, Revues des sciences fondamentales et appliquées, centre universitaire d'Oued Souf, $\mathrm{N}^{\mathrm{o}} 1$, 2009, pp.80-103. 
[10] Ghomri, A., Riguet, F. , Debabeche, M. Riguet, F, Journal of Fundamental and Applied Sciences, Université Hamma Lakhdar El Oued, No 4(2), 2012, pp.1-15.

[11] Hager, W.H., Wanoschek, R (1989), Hudraulic jump in trapezoidal channel, J. hydraulic research, Vol. 27, 1989, N.3.

How to cite this article:

Ghomri A, Riguet F, Khechana S. Contribution to the experimental study of the hydraulic jump in trapezoidal channel with positive slope. J. Fundam. Appl. Sci., 2018, 10(1), 191-200. 